\title{
Context-awareness for heterogeneous access management
}

\author{
C. Mannweiler, A. Klein, J. Schneider, and H. D. Schotten \\ Chair for Wireless Communications and Navigation, University of Kaiserslautern, Germany
}

\begin{abstract}
The increasing availability of both static and dynamic context information has steadily been driving the development of context-aware communication systems. Adapting system behavior according to current context of the network, the user, and the terminal can yield significant end-toend performance improvements. In this paper, we present a concept for how to use context information, in particular location information and movement prediction, for Heterogeneous Access Management (HAM). In a first step, we outline the functional architecture of a distributed and extensible context management system (CMS) that defines the roles, tasks, and interfaces of all modules within such a system for large-scale context acquisition and dissemination. In a second step, we depict how the available context information can be exploited for optimizing terminal handover decisions to be made in a multi-RAT (radio access technology) environment. In addition, the utilized method for predicting terminal location as well as the objective functions used for evaluating and comparing system performance are described. Finally, we present preliminary simulation results demonstrating that HAM systems that include current and future terminal context information in the handover decision process clearly outperform conventional systems.
\end{abstract}

\section{Introduction}

Context awareness has been introduced to a multitude of systems in order to adaptively control system behavior. Today, context information is made available for a broad variety of applications, for instance context-aware services for mobile terminals. These services increasingly rely on data gathered by terminal sensors, (wireless) sensor and actuator networks deployed in public places or indoor environments (Schnei-

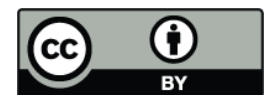

Correspondence to: C. Mannweiler (mannweiler@eit.uni-kl.de) der, 2009). Sensor data is complemented by information retrieved from the World Wide Web (such as social networks) or stored in databases. Classification and inference algorithms are used to semantically augment and transform data to higher levels of abstraction. The processed data can then be exploited by actuator networks, for adaptive system behavior, or for context-aware service creation and delivery. In a similar way, user and network context can be used for optimizing Heterogeneous Access Management (HAM) systems.

The growing need for wireless Internet access in combination with a more diverse landscape of radio access technologies (WLAN, GPRS, UMTS, HSDPA, WiMAX, etc.) as well as the demand for high data rates in the mobile domain increases the relevance of these systems. From a user perspective, they shall provide features such as "always on" and "always best connected". Operators are looking for solutions that more precisely allow charging and billing customers according to their actual resource consumption, thus more effectively controlling system utilization. In order to facilitate the achievement of these objectives, HAM systems have to be able to exploit not only radio network parameters but any available context information of the users. Systems using both network context information (such as network utilization or link capacity) and user context information (such as location or movement variables) are also called intelligent radio network access (IRNA) systems (Klein, 2009).

However, these systems impose strong constraints with regard to the quality of context. For instance, network selection decisions are very delay-sensitive. A distributed context management system for large-scale context acquisition and dissemination as well as accounting for quality of context with respect to availability, accuracy, delay, relevance, and confidence is a strict requirement for robust HAM. Reflecting these issues, the paper is organized as follows. Section 2 gives an overview on related work, Sects. 3 and 4 present a horizontal context management system with open interfaces and a HAM concept including a mechanism for

Published by Copernicus Publications on behalf of the URSI Landesausschuss in der Bundesrepublik Deutschland e.V. 
context prediction, respectively. In Sect. 5, we present preliminary simulation results that allow for a comparison of context-aware and conventional HAM. A conclusion and an outlook on future work is given in Sect. 6.

\section{State of the art and related work}

Context-aware network access management brings together two areas of research. On the one hand, Heterogeneous Access Management enabling intelligent access has become very relevant in a world that increasingly relies not only on being "online" permanently, but also on guaranteeing a highspeed network connection. On the other hand, context awareness is a key feature of systems that have to intelligently decide how to manage network resources.

\subsection{Network selection and heterogeneous access management}

In a more and more fragmented and heterogeneous wireless network landscape and with an increasing demand for high data rates, the need for efficient network access management across different radio access technologies (RATs), referred to as "Heterogeneous Access Management", becomes eminent. Radio resources are of course limited due to physical, technology-specific and regulatory constraints. Context, either provided by terminals, network nodes or from sensors deployed in the user's environment, may be utilized to significantly reduce the waste of scarce radio resources and efficiently manage wireless access across heterogeneous RATs.

The issue of network selection in an environment of diverse wireless access possibilities is widely addressed in today's research. HAM describes a concept for controlling radio resource allocation and utilization, as e.g. bandwidth, power, etc., across various RATs, where the main goals are to optimize overall system performance and to enable seamless mobility. HAM decisions and actions may be influenced by network operator policies (Taha et al., 2004), service level agreements (Yang et al., 2005), user preferences (Ormond et al., 2005), user location information (Pawar et al., 2009) or as a result of sophisticated resource utilization analysis (Blau et al., 2007). Further, the integration of a multitude of wireless access networks under a cross-layer context-aware architecture (Hasswa et al., 2007) has shown to increase user satisfaction and network throughput.

Inherently related to HAM is the issue of when to change the user's point of attachment to a specific network to another network due to limited resources in the former one, also referred to as "handover". Hence, the availability of network context is a prerequisite for efficient handover decisions. Moreover, it is beneficial to consider additional context information. However, the afore mentioned approaches exhibit no feature for incorporating movement prediction for network adaptation and HAM decisions.

\subsection{Context awareness}

The notion of context awareness can be found in manifold variations in different research areas of computer science. On the one hand, context consumers, such as context-aware applications and services, require context information in order to adapt to changes in their environment. On the other hand, context providers, e.g. sensors and (wireless) sensor networks, are key components for detecting the requested context. Moreover, GPS modules, temperature, ambient light, and acceleration sensors are becoming widespread with the recent success of smartphones and other mobile devices. For categorization of context sources, Chen (2004) proposes the following classes:

1. Direct access to sensors - Sensors deployed on network terminals can collect environmental context such as location or temperature as well as network context information.

2. Middleware infrastructure - The introduction of middleware infrastructure aims at strictly separating the processes of context acquisition and context management. The separation improves system extensibility and reusability.

3. Context server - In this approach, a resource-rich context server takes over the task of administering context data that it receives from various context sources. It relieves sensors and terminals from managing context requests from other entities.

Context management systems acquire, process, manage, and distribute context information according to the specific needs of applications and services. Exploiting the available context information for improving network performance is a meaningful application beyond conventional end consumer services such as location-based services. However, in both cases, a context management system with open and standardized interfaces is required in order to successfully disseminate context in a large scale. Many of the early contextaware systems in science have just been location-aware and were based on vertically integrated concepts. In the 1990s, location-aware tourist guide systems were developed that replaced or complemented traditional tourist guides (Abowd et al., 1997; Sumi et al., 1998; Cheverst et al., 2000). Meanwhile, there have been improvements with regard to both architectural frameworks for context-aware systems and the diversity and quality of available context information (e.g. advanced positioning technology (Jo et al., 2007)). European projects such as SPICE (Zhdanova et al., 2006) and MobiLife (Floréen et al., 2005) have set milestones in integrating context awareness functions into their respective architectures. Figure 1 depicts the C-CAST context management architecture with its split into relevant functions. Other authors, such as Chen (2004), have proposed agent-based approaches. 


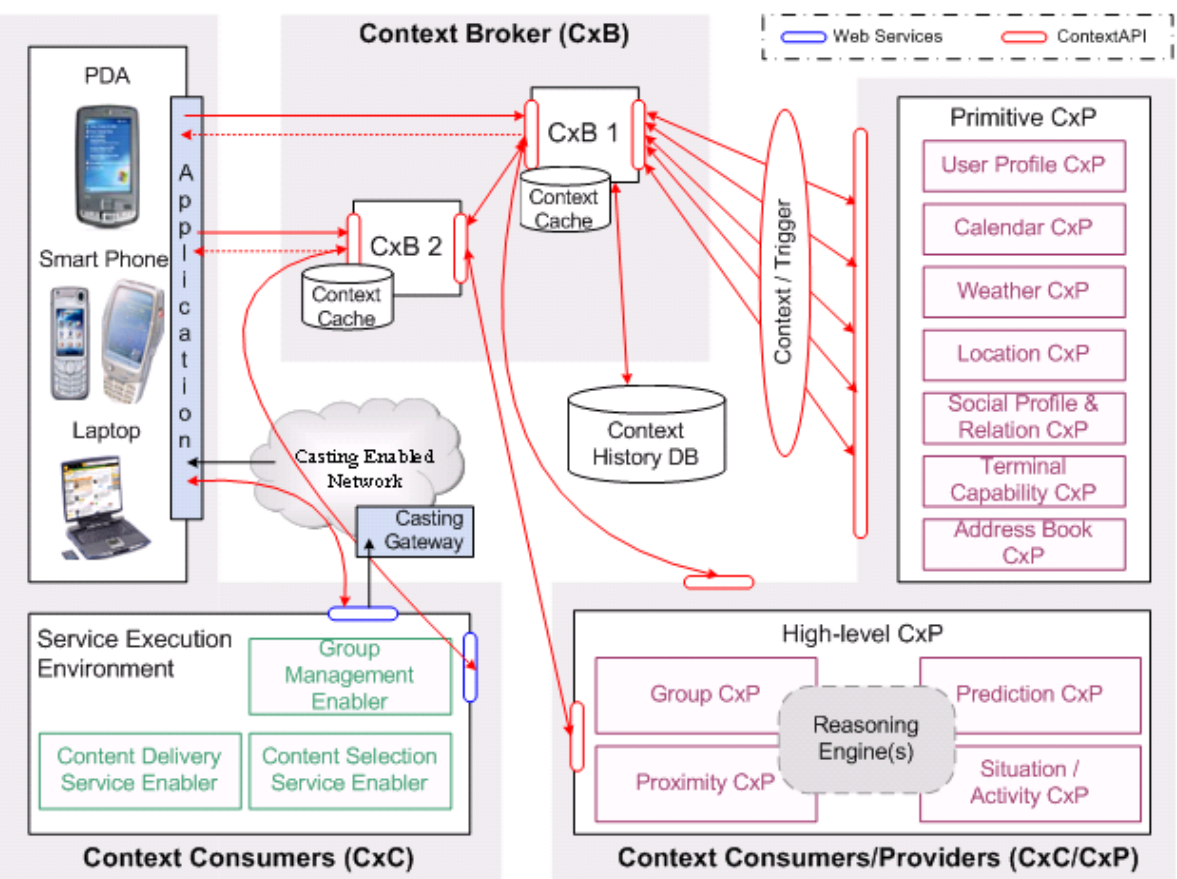

Fig. 1. C-CAST context management architecture.

However, all of these frameworks miss a comprehensive concept for a vertically disintegrated structure that facilitates the acquisition and dissemination of context information across different domains. In contrast, the proposed context management system disposes of open interfaces and a uniform context representation schema.

\section{A context management system for HAM}

Ideally, context management systems should be agnostic with respect to their application. However, depending on the intended utilization of context information, context management concepts have to comply with different requirements. In this section, we propose a context management system (CMS) that is based on the producer-consumer role model that can frequently be found in the area of context management, e.g. Pils et al. (2007). The C-CAST CMS as depicted in Fig. 1 is designed to acquire, manage, and distribute context information and to control the context quality required for HAM purposes. This functionality requires the following core architectural components: Context Providers, Context Brokers, and Context Consumers. Two different communication modes are available within the system. In the asynchronous mode, context is published if a specified condition or event comes true ("subscribe/publish mode"). In the synchronous mode, a request for context information is instantly answered ("request/provide mode").

\subsection{Context provider}

Context Providers (CxP) gather data from a collection of sensors, network, services (e.g. web services) or other relevant sources. A CxP employs various filtering, aggregation and reasoning mechanisms to infer context from raw sensor or other source data. This created context is then modeled according to a specified representation schema. Each provider is tailored to provide a particular type of context e.g. Calendar Provider provides calendar information about an entity and a Location Provider provides location information about an entity. Every CxP registers its availability and capabilities by sending appropriate announcements to the CMA and exposes interfaces to provide context information to the CMA.

\subsection{Context broker}

Context Brokers $(\mathrm{CxB})$ take on the role of middlemen. Their main functionality is to maintain a distributed registry of available CxPs and their capabilities based on the announcement sent by CxPs. Based on that registry, they can provide a CXP look-up service to entities searching for certain context data. The distributed structure of the registry needs to be maintained constantly in order to have an even distribution of information.

\subsection{Context consumer}

A Context Consumer $(\mathrm{CxC})$ is an architectural component that uses context data, for example a context based 
application or a network management entity. A CxC can retrieve context information by sending a request to the system, i.e. by directly invoking a $\mathrm{CxP}$ over a generic interface. Another way for the $\mathrm{CxC}$ to obtain information is to subscribe to a specific context information. It will be notified by a $\mathrm{CxP}$ as soon as the specified notification condition materializes.

\section{A concept for heterogeneous access management}

The heterogeneity of today's wireless access possibilities imposes challenges for efficient access and resource management across different RATs. The proposed HAM concept, as shown in Fig. 2, accounts for the characteristics and status of each RAT while simultaneously trying to accommodate users, who face different environmental conditions, with the best possible end-to-end performance.

Since the use of context information enriches the information basis on which HAM decisions are taken, network selection and handover decisions will be significantly improved, given context such as network load and user movement predictions is available.

Further, the a-priori knowledge of available access networks within reach will significantly reduce terminal scan times for access networks, yielding decreased terminal power consumption. Multicasting streams will be efficiently rerouted and transmitted knowing terminal capabilities and points of attachment (Janneteau et al., 2009). Mechanisms of modern wireless RATs as e.g. link adaptation will also benefit from detailed context information such as user environment and history information of certain hotspots.

Since each wireless access network provides a different level of Quality of Service (QoS), capacity, and coverage, various parameters are accounted for to ensure satisfying end-to-end performance. Relevant parameters are either static (e.g. system bandwidth) or dynamically changing due to users' movements and environmental conditions (e.g. received signal strength). While static parameters will be retrieved from databases, dynamic parameters need to be measured, monitored and assessed.

In case that $n$ real-valued, non-negative context scopes are available for HAM, we can denote their values at time $t$ as $x_{1, t}, x_{2, t}, \ldots, x_{n, t}$ where each of the scopes can lie within an interval $\left[l_{k}, u_{k}\right], k=1,2, \ldots, n$ that is relevant for HAM decisions. Values lying outside their respective interval have to be considered separately. In order to normalize different context scopes to the interval $[0,1]$, each $x_{k, t}$ is divided by the respective interval width $u_{k}-l_{k}$ :

$x_{k, t}^{\prime}=\frac{x_{k, t}-l_{k}}{u_{k}-l_{k}}$

In the domain of Heterogeneous Access Management, the majority of context scopes have to be considered per user (or terminal) and base station since they describe a characteristic of the connection between the terminal and the base station. An example would be the signal strength of different WLAN access points detected by different user terminals. Hence, it is necessary to more specifically denote context values with two additional indexes $i$ and $j$, where $i$ represents the terminal and $j$ the base station. However, scopes exist that fulfill $x_{i, j, k, t}=x_{j, k, t}$, i.e. context scopes being equal for all i, e.g. the current number of users attached to an access point. Moreover, we can weigh the normalized context values according to their relevance $\left(r_{i, j, k} \in[0,1]\right)$ for the evaluation of the (possible) connection and their confidence level $\left(c_{i, j, k} \in[0,1]\right)$. In conjunction with Eq. (1), this yields

$x_{i, j, k, t}^{\prime \prime}=c_{i, j, k} \cdot r_{i, j, k} \cdot \frac{x_{i, j, k, t}-l_{i, j, k}}{u_{i, j, k}-l_{i, j, k}}$

For decisions related to HAM, it is crucial to find (based on available context information) the best radio connection for a single terminal, taking into account overall system performance. Hence, for every point in time $t$, the quality $q(j)$ of each (possible) connection to the available point of attachments $j$ (base stations, access points, etc.) is evaluated by calculating

$q_{i, t}(j)=\sum_{k=1}^{n} x_{i, j, k, t}^{\prime \prime \prime}$,

where $x_{i, j, k, t}^{\prime \prime \prime}=x_{i, j, k, t}^{\prime \prime}$ if $x_{k, t}^{\prime}$ is positively correlated to the evaluation of the possible connection, i.e. an increasing value of $x_{k, t}^{\prime}$ yields a high-rated connection (e.g. received signal strength), and $x_{i, j, k, t}^{\prime \prime \prime}=1-x_{i, j, k, t}^{\prime \prime}$ if $x_{k, t}^{\prime}$ is negatively correlated to the evaluation of the possible connection, i.e. a decreasing value of $x_{k, t}^{\prime}$ yields a higher rating (e.g. distance to point of attachment). For every terminal $i$, the maximum $q_{i, t, \max }$ of all $q_{i, t}(j)$ identifies the (theoretically) best point of attachment to be connected to. However, this does not take into account cost associated to an executed handover (such as terminal battery power, use of network resources, etc.). If $j=j_{0}$ denotes the point of attachment for the currently active connection of the terminal, we define a margin $y_{i, j}$ by which $q_{i, t, \max }$ has to exceed $q_{i, t}\left(j_{0}\right)$. Thus, the handover is only performed if

$q_{i, t, \max }>q_{i, t}\left(j_{0}\right)+y_{i, j}$.

The margin $y_{i, j}$, besides ensuring a better connection quality in absolute terms, allows for trading off handover costs against enhanced connection quality, where handover costs are not limited to signaling costs but also comprise costs spent for acquiring, processing, and evaluating context information.

Further, in order to beneficially adapt to the dynamics described by this linear, terminal-centric model, gathered context information must fulfill certain requirements in terms of context quality, where relevant quality criteria are availability, accuracy, topicality, delay, relevance and confidence. Thus, the use of context information for optimizing HAM 


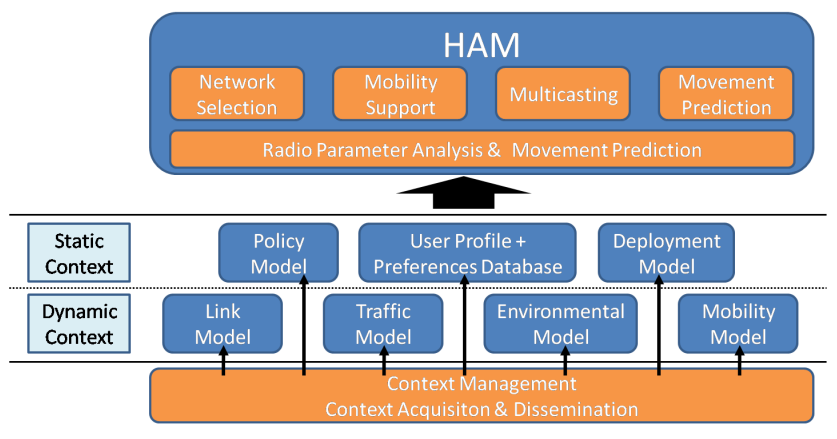

Fig. 2. Concept for heterogeneous access management.

and system performance requires quality control and management of the acquired context information. The aim of the proposed context management architecture therefore is to adaptively control and manage context information according to the requirements of different Context Consumers, e.g. the HAM module as depicted in Fig. 2.

\section{Simulation results}

For a quantitative verification of the outlined concept, a context-aware radio network simulator with the following key characteristics has been implemented. The overall deployment can be set up flexibly by selecting cell number, structure (hexagonal, either one (centrally positioned) BS per cell or one BS serving three cells via directed antennas) and layout. Base stations can have an off-set to one another and can (depending of RAT) vary in their transmission power. The number of terminals can be set up flexibly and remains constant during simulation time. Different speed classes can be attributed to every single terminal and, depending on the assigned speed, a terminal performs a defined form of random walk. Depending on a set of analyzed parameters (distance to BS, idle BS capacity, geometry factor, etc.), a terminal is assigned to the best ranked BS. One simulation time step is $10 \mathrm{~ms}$.

In order to evaluate the influence of available context information, a movement prediction method is integrated into the simulation and considered for HAM decisions. The used prediction algorithm calculates the possible terminal locations 50 simulation steps in advance using a simplified prediction model based on the "dead reckoning" method. Further, using the estimated location and knowing the location of base stations in the vicinity, the terminal evaluates its geometry factor (Holma et al., 2007) to available (and active) base stations around. The evaluation process only foresees a handover execution when the estimated geometry of the respective terminal exceeds a predefined threshold (e.g. usual handover margin for UMTS 3-10 dB). If these conditions are met, handover execution is triggered. For an initial compar-

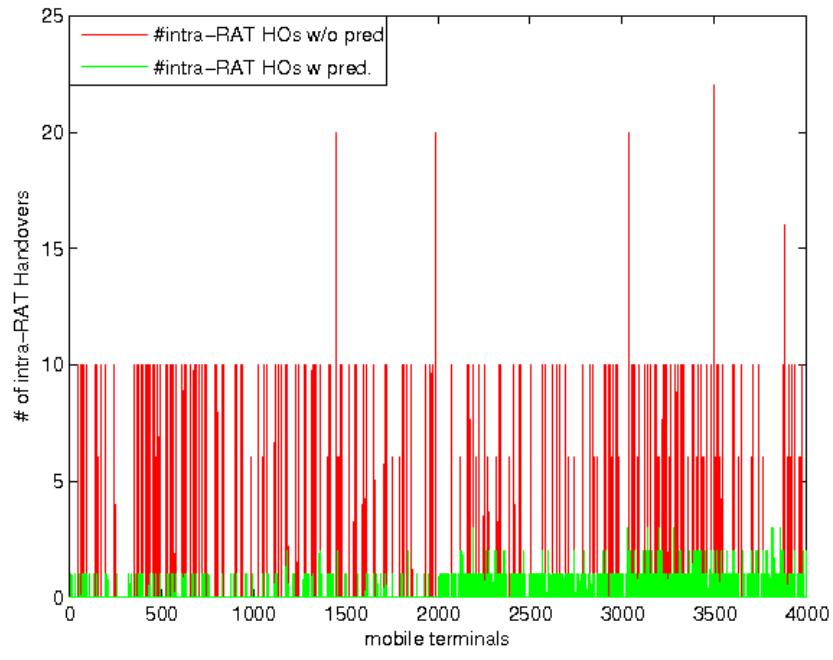

Fig. 3. Number of Intra-RAT handovers for simulated terminals.

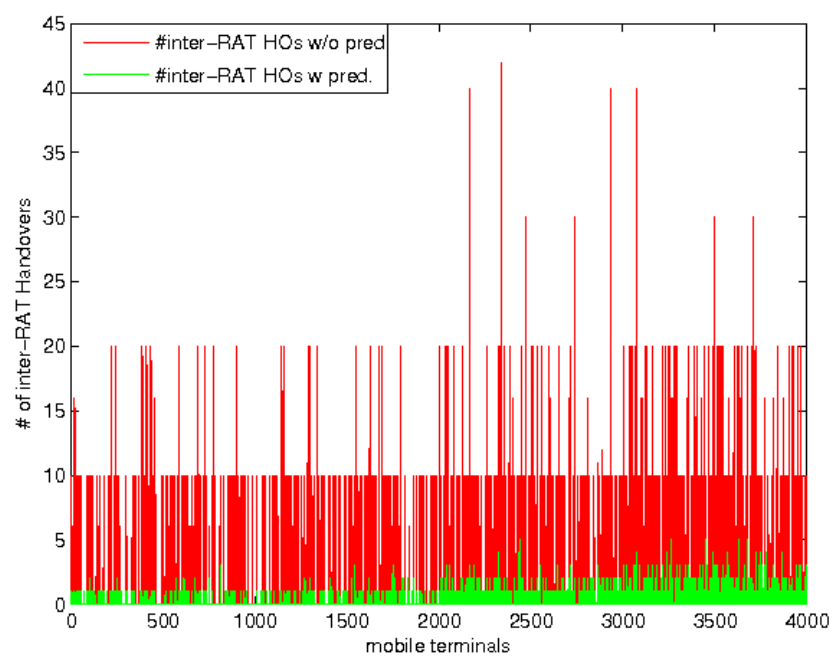

Fig. 4. Number of Inter-RAT handovers for simulated terminals.

ison of HAM performance with and without movement prediction, the number of performed handovers is considered. In general, a lower number is preferable since a handover is costly in terms of resource utilization. Figures 3 and 4 depict the amount of inter-RAT and intra-RAT handovers for 4000 terminals.

The comparison of inter-RAT and intra-RAT handover counters shows a significantly higher number of handovers being executed in the scenario without movement prediction (red graph) compared to the case where the prediction algorithm is incorporated (green graph). Although only preliminary and only including specific context information, the results clearly demonstrate the potential of context-aware HAM. 


\section{Conclusions}

HAM is one of the key future research areas for optimizing radio network performance. This paper presented a framework for the integration of context information into Heterogeneous Access Management (HAM). A formal method assessing link quality based on available context information has been developed for triggering handover mechanisms. The proposed Context Management Architecture is responsible for large-scale context acquisition and dissemination. Finally, based on the outlined HAM concept, we presented a context-aware radio network simulator that is capable of modeling manifold mobile communications scenarios. First results show that the availability of current and predicted context information yields significant performance improvements in HAM. Although only preliminary, these findings demonstrate the potential of context-awareness for HAM. Further research activities include a more detailed analysis of the impact of context information availability on simulation results beyond the preliminary findings. In order to draw more valid conclusions, additional context models will be implemented within the simulator, the aim being, among other, to separate context of major influence from that of low or medium influence on system performance.

Acknowledgements. This work has been partially supported by the Collaborative Project Context Casting (C-CAST - Provide an End-to-End Context-Aware Communication Framework), which is funded by the European Commission under the Seventh Framework Programme.

\section{References}

Abowd, G. D., Atkeson, C. G., Hong, J., Long, S., Kooper, R., and Pinkerton, M.: Cyberguide: a mobile context-aware tour guide, Wireless Networks, 3(5), 421-433, 1997.

Blau, I., Wunder, G., Karla, I., and Sigle, R.: Cost Based Heterogeneous Access Management in Multi-Service, Multi-System Scenarios, The 18th Annual IEEE International Symposium on Personal, Indoor and Mobile Radio Communications (PIMRC'07), Athens, 2007.

Chen, H.: An Intelligent Broker Architecture for Pervasive ContextAware Systems, University of Maryland, College Park, MD, 2004.

Cheverst, K., Davies, N., Mitchell, K., Friday, A., and Efs-tratiou, C.: Developing a context-aware electronic tourist guide: some issues and experiences, in: Proceedings of the SIGCHI conference on Human Factors in Computing Systems, The Hague, 2000.

Floréen, P., Przybilski, M., Nurmi, P., Koolwaaij, J., Tarlano, A., Wagner, M., Luther, M., Bataille, F., Boussard, M., Mrohs, B., and Lau, S.: Towards a Context Management Framework for MobiLife, 14th IST Mobile and Communications Summit, Dresden, 2005.

Hasswa, A., Nasser, N., and Hassanein, H.: A seamless contextaware architecture for fourth generation wireless networks, Wireless Personal Communications, Springer, Heidelberg, 43, 10351049, 2007.
Holma, H., Reunanen, J., Chan, L., Mogensen, P., Pedersen, K., Horneman, K., Vihriälä, J., and Juntti, M.: Physical Layer Performance, in: WCDMA for UMTS : HSPA Evolution and LTE, edited by: Holma, H. and Toskala, A., Chichester, England, 2007.

Janneteau, C., Simoes, J., Antoniou, J., Christophorou, C., Kellil, M., Klein, A., Neto, A., Pinto, F. C., Roux, P., Sargento, S., Schotten, H. D., and Schneider, J.: Context-aware Multiparty Networking, Conference Proceedings of ICT MobileSummit, Santander, 2009.

Jo, D., Lee, J., Lee, S., Ha, T., Kwon, T., and Choi, Y.: Signal Dragging: Effects of Terminal Movement on War-Driving in CDMA/WCDMA Networks, in: LoCA 2007. LNCS, edited by: Hightower, J., Schiele, B., and Strang, T., Springer, Heidelberg, 4718, 211-227, 2007.

Klein, A., Mannweiler, C., Schneider, J., and Schotten, H.: A Framework for Intelligent Radio Network Access Based on Context Models, Proceedings of 22nd WWRF Meeting, Paris, 2009.

Ormond, O., Perry, P., and Murphy, J.: Network Selection Decision in Wireless Heterogeneous Networks. IEEE 16th International Symposium on Personal, Indoor and Mobile Radio Communications, Berlin, 2005.

Pawar, P., van Beijnum, B.-J., Wac, K., Hermens, H., and Konstantas, D.: Towards Location Based QoS-Aware Network Selection Mechanism for the Nomadic Mobile Services. IEEE Consumer Communications and Networking Conference, Special Session on Beyond GPS - Where Navigation meets Consumer Communications (CCNC'09), Las Vegas, NV, 2009.

Pils, C., Roussaki, I., Pfeifer, T., Liampotis, N., and Kalatzis, N.: Federation and Sharing in the Context Marketplace, in: LoCA 2007, edited by: Hightower, J., Schiele, B., and Strang, T., LNCS, Springer, 4718, 121-138, Heidelberg, 2007.

Schneider, J., Mannweiler, C., Klein, A., and Schotten, H.: Erfassung von Umgebungskontext und Kontextmanagement, 14. ITG Fachtagung Mobilkommunikation, Osnabrück, 2009.

Sumi, Y., Etani, T., Fels, S., Simonet, N., Kobayashi, K., and Mase, K.: C-map: Building a context-aware mobile assistant for exhibition tours, in: Community Computing and Support Systems, Social Interaction in Networked Communities, edited by: Ishida, T., LNCS, Springer, Heidelberg, 1519, 137-154, 1998.

Taha, A.-E. M., Hassanein, H. S., and Mouftah, H. T.: On Robust Allocation Policies in Wireless Heterogeneous Networks. Proceedings of the First International Conference on Quality of Service in Heterogeneous Wired/Wireless Networks (QSHINE'04), 2004.

Yang, X., Bigham, J., and Cuthbert, J.: Resource Management for Service Providers in Heterogeneous Wireless Networks, Wireless Communications and Networking Conference, New Orleans, LA, 2005.

Zhdanova, A. V., Zoric, J., Marengo, M., Van Kranenburg, H., Snoeck, N., Sutterer, M., Räck, C., Droegehorn, O., and Arbanowski, S.: Context Acquisition, Representation and Employment in Mobile Service Platforms, 15th IST Mobile and Wireless Communications Summit, Myconos, 2006. 